\title{
The design and performance of the ATLAS Inner Detector trigger for Run 2 LHC collisions at $\sqrt{s}=13$ TeV
}

\author{
Fabrizio Miano* on behalf of the ATLAS Collaboration ${ }^{\dagger}$ \\ University of Sussex \\ E-mail: fabrizio.miano@cern.ch
}

\begin{abstract}
The Run 2 of the Large Hadron Collider presents challenging high rate conditions for data processing and analysis within the ATLAS trigger system. The ATLAS Inner Detector trigger implements the algorithms used for identification of tracks for all physics signatures within the ATLAS trigger. The ID trigger was updated and redesigned during the 2013-2015 Long Shutdown to meet the challenging conditions of Run 2. As well, for Run 2, a new pixel detector layer was added in very close proximity to the beam pipe to enhance the ID trigger performance. The redesigned ID trigger algorithms for Run 2 are described, illustrating the significant improvements gained by the new tracking strategies adopted to deal with the increased rate. The performance of the ID trigger in Run 2 is shown in terms of efficiency and resolution, using data collected from the 25 ns bunch spacing data collected in 2015. The ID trigger continues to show excellent performance, with efficiencies greater than $99 \%$.
\end{abstract}

38th International Conference on High Energy Physics

3-10 August 2016

Chicago, USA

\footnotetext{
*Speaker.

${ }^{\dagger}$ Special thanks to Dr Mark Sutton for having thoroughly supervised my work.
} 


\section{Introduction: the ATLAS detector and the trigger}

The ATLAS experiment at the LHC [1,2] operates under extremely demanding conditions. The challenging conditions due to the upgrade of the machine required a significant upgrade of the ATLAS detector and trigger systems, which have been performed during the first Long Shutdown (LS1) between LHC Run 1 and Run 2. In particular, the tracking and the trigger system, being essential and used to determine the events to be recorded to offline storage, were upgraded in order to cope with higher rates and to perform a very intense and diverse physics programme. The Inner Detector (ID) trigger provides track and vertex reconstruction used to determine whether an event should be read out or discarded. In Run 1, the ID trigger systems provided excellent performance in physics reconstruction; the same high standard must be retained during Run 2.

ATLAS is a general purpose detector with a cylindrical symmetry and an almost full solid angle coverage around the interaction point. The main sub-systems of ATLAS are the ID, nearest the interaction region used to reconstruct charged particle tracks used in the selection of physics objects for all trigger signatures, the calorimeter, and the Muon System (MS). A new innermost pixel layer, the insertable B-layer (IBL), was added during LS1 around a new and thinner beam pipe. The IBL allows more robust track reconstruction, with better impact parameter resolution and more precise vertex reconstruction. In Run 1, the trigger consisted of a hardware-based Level 1 (L1) trigger which identifies Regions of Interest (RoIs) which contain features that merit further processing in the later trigger levels, a fast software-based Level 2 (L2) able to read out the full granularity detector element within the RoIs identified by L1, and a software-based Event Filter (EF) trigger that used more offline reconstruction algorithms. For the LHC Run 2 the computational architecture of the ATLAS trigger has been considerably redesigned. The two software-based levels were combined into a single High Level trigger (HLT). Running the full HLT reconstruction for each event on an individual node, affords the opportunity to better optimise the RoI geometry and use an advanced multi-stage strategy for the tau and $b$-jet triggers. In Run 2 an advanced multistage approach, reducing the detector volume of RoI, is used for the tracking of taus and $b$-jets. The first stage is to identify leading tracks within a long in $z$ but narrow in $\eta$ and $\phi$ RoI running the Fast Track Finder (FTF) algorithm. The leading tracks are used to construct a second-stage RoI, constrained in both $\eta$ and $\phi$, but very tightly constrained in polar angle and with a small $z$ position width. The FTF is then run again in this wider second stage RoI, followed by the Precision Tracking. This allows faster execution of the tracking algorithms since they run in a significantly reduced volume with respect to Run 1 .

\section{Performance results from Run 2 data}

Figure 1 shows the tracking efficiency with respect to offline tracks for the $24 \mathrm{GeV}$ tight electron trigger. The tracking efficiency is measured with respect to offline tracks with $p_{\mathrm{T}}>20 \mathrm{GeV}$ from the electron support trigger, which does not use the trigger tracks in the selection, but is otherwise identical to the physics trigger. The closest trigger track within a cone of $\Delta R=\sqrt{\Delta \eta^{2}+\Delta \phi^{2}}<$ 0.05 of the offline reconstructed track is selected as the matching trigger track and the efficiency is shown as a function of the $\eta$ and of the $p_{\mathrm{T}}$ of the offline track. The Fast Track Finder efficiency integrated over $p_{\mathrm{T}}$ is better than $99 \%$ for all pseudorapidities. The $\sim 0.5 \%$ inefficiency observed at low $p_{\mathrm{T}}$ is due to bremsstrahlung energy loss by electrons. 

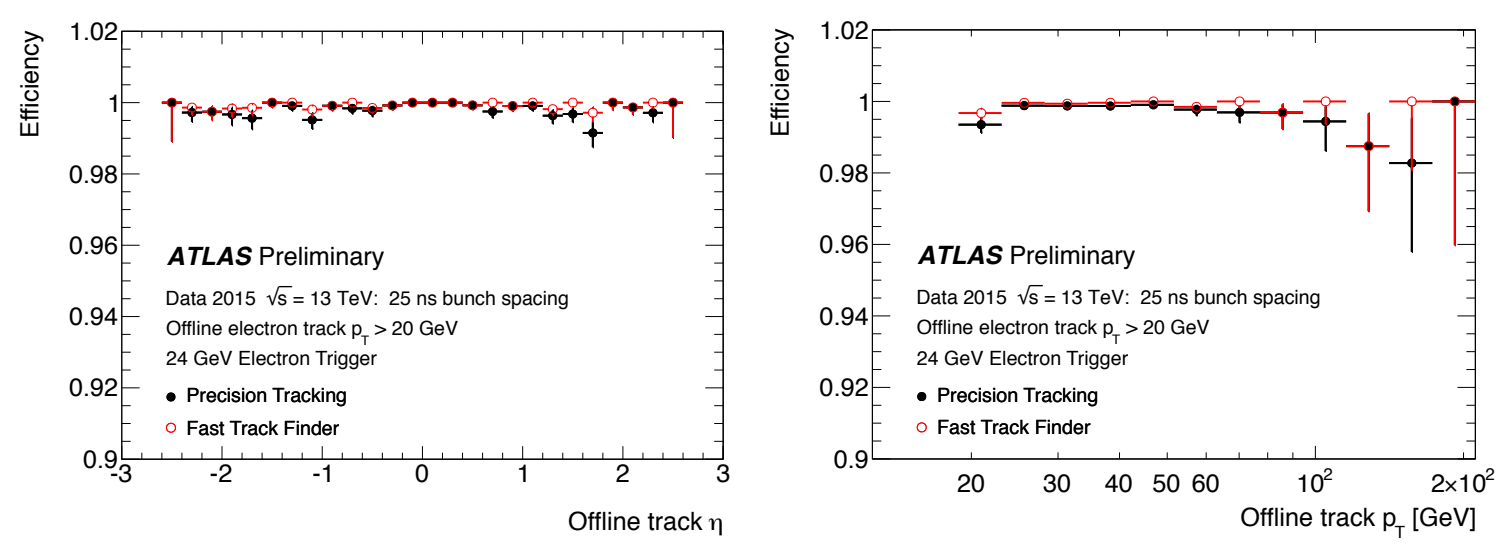

Figure 1: The ID tracking efficiency for the $24 \mathrm{GeV}$ electron trigger; versus the offline electron pseudorapidity (left) and the offline electron $p_{\mathrm{T}}$ (right). Bayesian uncertainties are shown[3].

Figure 2 shows the performance of the muon tracking in the ID trigger with respect to loosely selected offline muon candidates with $p_{\mathrm{T}}>6 \mathrm{GeV}$. Figure 2 shows the efficiency as a function of the offline muon transverse momentum. The efficiency is effectively $100 \%$ for all $p_{\mathrm{T}}$ for both FTF and Precision Tracking. Also shown is the resolution with respect to the offline tracks of the transverse track impact parameter as a function of the offline muon $p_{\mathrm{T}}$. The resolution in the Precision Tracking is better than $15 \mu \mathrm{m}$ for muon candidates with offline $p_{\mathrm{T}}>20 \mathrm{GeV}$.
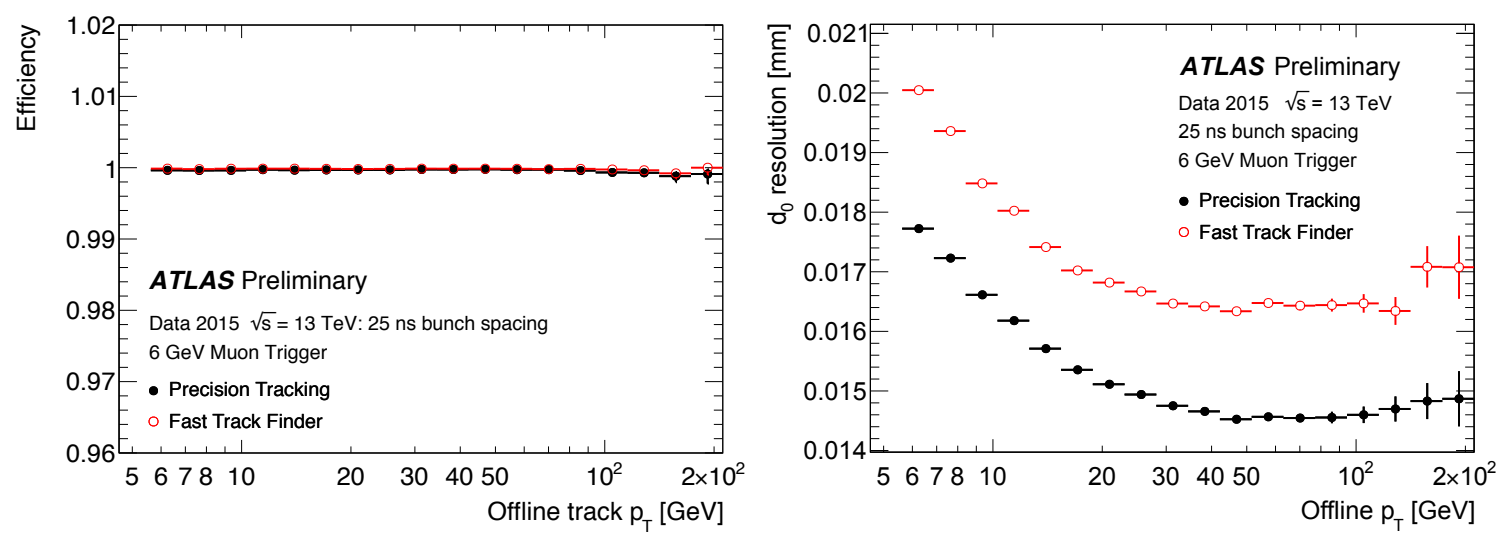

Figure 2: The ID tracking performance from the muon trigger with $p_{\mathrm{T}}>6 \mathrm{GeV}$; the efficiency versus the offline muon $p_{\mathrm{T}}(\mathrm{left})$, the resolution on the transverse impact parameter, $d_{0}$ versus offline muon $p_{\mathrm{T}}$ (right). Bayesian uncertainties are shown[3].

For $b$-jet tracking a multi-stage tracking strategy has been adopted. The first stage vertex tracking takes all jets identified by the jet trigger with $E_{\mathrm{T}}>30 \mathrm{GeV}$ and reconstructs tracks using the FTF in a narrow region in $\eta$ and $\phi$ around the jet axis for each jet, but with $|z|<225 \mathrm{~mm}$ along the beam line. So found tracks are used to reconstruct the primary vertex position. This vertex is used to define wider RoIs around the jet axes, with $|\Delta \eta|<0.4$ and $|\Delta \phi|<0.4$ but with $|\Delta z|<20 \mathrm{~mm}$ relative to the primary vertex $z$ position. These RoIs are then used for the second stage reconstruction that runs the FTF in the wider $\eta$ and $\phi$ regions followed by the Precision Tracking, secondary vertex, originating from $b$ hadron decays, and $b$-tagging algorithms. 

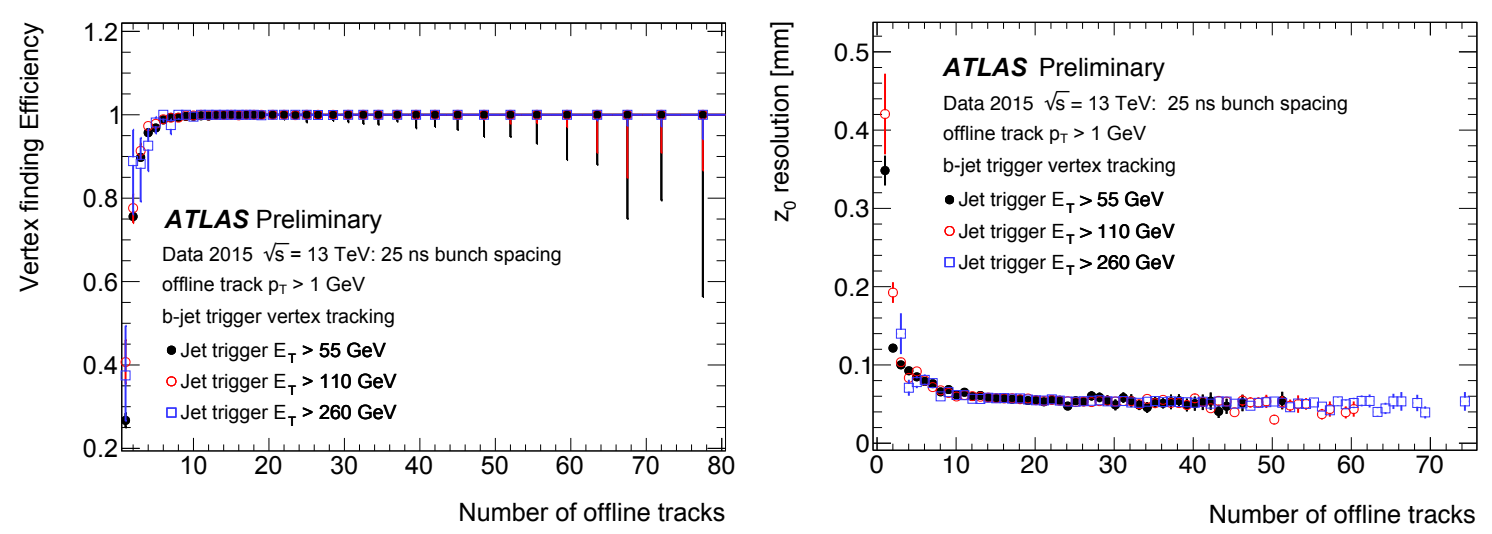

Figure 3: The trigger vertexing performance in the $b$-jet signatures for 55,110 and $260 \mathrm{GeV}$ jet triggers; the vertexing efficiency (left) as a function of the number of offline tracks within the jets used for the vertex tracking, the vertex $z$-resolution with respect to offline (right)[3].

The performance of the primary vertexing in the $b$-jet vertex tracking can be seen in Figure 3 which shows the vertex finding efficiency with respect to offline vertices in jet events with at least one jet with transverse energy above 55,110 , or $260 \mathrm{GeV}$ and with no additional $b$-tagging requirement. The efficiency (left) is shown as a function of the number of offline vertex tracks with $p_{\mathrm{T}}>1 \mathrm{GeV}$ that lie within the boundary of the wider RoI - as mentioned before for $b$-jet from the selected jets. The efficiency rises sharply and is greater than $90 \%$ for vertices with three or more tracks, and rises to more than $99.5 \%$ for vertices with five or more tracks. The resolution (right) in $z$ with respect to the offline $z$ position is better than $100 \mu \mathrm{m}$ for vertices with two or more reconstructable offline tracks and improves to $60 \mu \mathrm{m}$ for vertices with ten or more reconstructable offline tracks.

\section{Conclusions}

The ATLAS trigger was improved during LS1 and the ID trigger algorithms reimplemented. The design and performance of the ATLAS tracking trigger system redesigned for Run 2 has been presented. The performance of the Inner Detector tracking for electrons, muons and $b$-jets has been presented. Even with the more demanding Run 2 conditions the ID trigger performance is as good as, or better than Run 1 performance. The electron trigger efficiency is better than $98 \%$, the muon trigger efficiency is very close to $100 \%$, and the $b$-jet trigger has $100 \%$ efficiency of finding a $b$-jet vertex with at least 6 reconstructable tracks.

\section{References}

[1] L. Evans and P. Bryant, LHC Machine JINST 3 (2008) S08001. doi:10.1088/1748-0221/3/08/S08001

[2] ATLAS Collaboration, "The ATLAS Experiment at the CERN Large Hadron Collider", JINST 3 (2008) S08003. doi:10.1088/1748-0221/3/08/S08003

[3] ATLAS Collaboration, HLT Tracking Public Results, (2016), https://twiki.cern.ch/twiki/bin/view/AtlasPublic/HLTTrackingPublicResults 